Discrete Comput Geom 27:239-247 (2002)

DOI: $10.1007 / \mathrm{s} 00454-001-0059-2$

Geometry

\title{
On Asymmetry of Some Convex Bodies
}

\author{
Qi Guo* and S. Kaijser \\ Department of Mathematics, Uppsala University, \\ Box 480, S-751 06 Uppsala, Sweden \\ \{guo, sten\}@math.uu.se
}

\begin{abstract}
We determine Minkowski's measure of asymmetry for convex hulls of a point and some sets for which the asymmetry is known. Some properties of the asymmetry measure are found which may indicate some interesting properties of convex bodies.
\end{abstract}

\section{Notation and Definitions}

For general convex bodies (i.e., convex sets with non-empty interior) in $R^{n}$, several different kinds of measures of asymmetry (or symmetry) have been introduced to describe how nearly symmetric a convex body is (see [1], [2], [6], and [9]). As is known, although these definitions of measures of asymmetry are quite simple, and they do characterize some properties of convex bodies (see [4], [5], [7], and [8]), there is still not much known about these measures.

In this paper we consider mainly the Minkowski measure of asymmetry for certain convex bodies. We introduce the concept of the asymmetry function for a convex body, and we find some properties of these asymmetry functions which may indicate some interesting properties of convex bodies.

For convenience, we first recall some notation and definitions. We denote by $R^{n}$ the $n$-dimensional linear space (in this paper we always assume $n \geq 2$ ), by $C, D \subset R^{n}$ the convex bodies, by $d(\cdot, \cdot)$ a positively homogeneous and translation-invariant distance on $R^{n}$, and by $T$ an affine map on $R^{n}$.

Given a convex body $C \subset R^{n}$, for any $x \in \operatorname{int} C$, the set of interior points of $C$, and any hyperplane $H$ containing $x$, there exist two support hyperplanes $H_{1}, H_{2}$ of $C$ which are parallel to $H$ and lie on either side of $H$, respectively. Let $r(H, x)$ be the ratio (which

\footnotetext{
* Address in China: Department of Mathematics, Harbin Institute of Technology, 150001 Harbin, People's Republic of China.
} 
is independent of the distance $d$ on $R^{n}$ ), not less than 1, in which $H$ divides the distance between $H_{1}$ and $H_{2}$, then define $r_{x}=\sup \{r(H, x): H \ni x\}$. The Minkowski measure of asymmetry as $(C)$ of $C$ is defined by (see [6])

$$
\operatorname{as}(C)=\inf \left\{r_{x}: x \in \operatorname{int} C\right\} .
$$

Since a convex body can be determined by all its support hyperplanes, i.e., $C=$ $\left\{x:-1 \leq f(x) \leq 1, f \in F_{C}\right\}$ where $F_{C}$ is the set of all affine functions on $R^{n}$ which satisfy that both $\{x: f(x)=1\}$ and $\{x: f(x)=-1\}$ are support hyperplanes to $C$, so equivalently the Minkowski measure of asymmetry can also be defined by affine functions (see [8]), but here we state it as a lemma (the proof is trivial):

Lemma 1. Given a convex body $C \subset R^{n}$. For any $x \in \operatorname{int} C$,

$$
r_{x}=\sup _{f \in F_{C}} \frac{1-f(x)}{1+f(x)}
$$

hence

$$
\operatorname{as}(C)=\min _{x \in \operatorname{int} C}\left(\sup _{f \in F_{C}} \frac{1-f(x)}{1+f(x)}\right) .
$$

Remark 1. Generally for a non-constant affine function $f$, let $m_{f}=\min _{x \in C} f(x)$, $M_{f}=\max _{x \in C} f(x)$. If $F_{C}^{*}$ denotes a set of some affine functions which satisfies that $C=\left\{x: m_{f} \leq f(x) \leq M_{f}, f \in F_{C}^{*}\right\}$, then it is easy to see that

$$
r_{x}=\sup _{f \in F_{C}^{*}}\left(\frac{M_{f}-f(x)}{f(x)-m_{f}}, \frac{f(x)-m_{f}}{M_{f}-f(x)}\right) .
$$

A point $x$ satisfying $r_{x}=\operatorname{as}(C)$ is called a critical point (of $C$ ). For any $C$ there exists at least one critical point (see [6]), and for symmetric convex bodies, the center is the only critical point. It is known (see [9]) that $1 \leq \operatorname{as}(C) \leq n$, and as $(C)=1$ iff $C$ is symmetric, as $(C)=n$ iff $C$ is a simplex.

Now given a convex body $C \subset R^{n}$, for any $x \in R^{n}$, let $\hat{C}(x)=\operatorname{conv}(x, C)$ where conv denotes the convex hull, then we have the following:

Definition 1. For any convex body $C \subset R^{n}$, the function $A_{C}: R^{n} \rightarrow R$, defined by $A_{C}(x)=\operatorname{as}(\hat{C}(x))$ for any $x \in R^{n}$, is called the asymmetry function (of $C$ ). Especially when $C$ is symmetric and the center of $C$ is the origin, the asymmetry function of $C$ is denoted by $A_{C}^{0}(x)$.

Fact 1. If $x \in C$, then $A_{C}(x)=\operatorname{as}(C)$.

Fact 2. For any invertible affine map $T$ on $R^{n}, A_{C}(x)=A_{T(C)}(T(x)), x \in R^{n}$, especially $A_{C}^{0}(x)=A_{C}^{0}(-x)$. 
Fact 3. Given a convex body $C \subset R^{n}$ and $x \in R^{n}$, let $F_{C}^{1}=\left\{f: f \in F_{C},|f(x)| \leq 1\right\}$ and $F_{C}^{2}=\left\{f: f \in F_{C}, f(x) \geq 1\right\}\left(F_{C}^{2}\right.$ may be empty). Then for any $y \in \operatorname{int} \hat{C}(x)$, we have

$$
r_{y}=\max \left\{\sup _{f \in F_{C}^{1}} \frac{1-f(y)}{1+f(y)} \sup _{f \in F_{C}^{2}} \frac{f(x)-f(y)}{1+f(y)}, \sup _{f \in F_{C}^{2}} \frac{1+f(y)}{f(x)-f(y)}\right\},
$$

which is useful for computing $A_{C}(x)$.

Fact 4. For any convex body $C \subset R^{n}, A_{C}(x)$ is continuous (with respect to any distance $d$ on $\left.R^{n}\right)$, and $1 \leq A_{C}(x) \leq n$.

Facts 1 and 2 are trivial, Fact 3 comes from the remark following Lemma 1, that $1 \leq A_{C}(x) \leq n$ is from the property of the Minkowski measure of asymmetry, and continuity follows from standard compactness arguments.

\section{Asymmetry Functions of Symmetric Convex Bodies}

In this section we consider asymmetry functions of (centrally) symmetric convex bodies only, and we assume that the center of the symmetric convex bodies is at the origin. For such a symmetric convex body $C$, denote by $B_{C}$ the Banach space $R^{n}$ with $C$ as its unit ball, by $B_{C}^{\prime}$ the dual space of $B_{C}$, by $S\left(B_{C}^{\prime}\right)$ the unit sphere of $B_{C}^{\prime}$, and by $\|\cdot\|_{C}$ (or simply $\|\cdot\|)$ the norm on $B_{C}$.

It is clear that for these symmetric convex bodies $C, S\left(B_{C}^{\prime}\right)=F_{C}$, i.e., $F_{C}, F_{C}^{1}$, and $F_{C}^{2}$ consist of linear functionals. Now we have our following main result.

Theorem 1. For any convex body $C \subset R^{n}$ with the origin o as its center, we have:

(1) $A_{C}^{0}(x)=\max \{1,2\|x\| /(\|x\|+1)\}, x \in R^{n}$.

(2) For any $x \in R^{n}$, the critical point $x^{*}$ of $\hat{C}(x)$ is unique, and

$$
x^{*}=\max \left\{0, \frac{\|x\|-1}{3\|x\|+1}\right\} x .
$$

Proof. If $\|x\| \leq 1$, then $A_{C}^{0}(x)=\operatorname{as}(C)=1$, and $x^{*}=o$. Now let $\|x\|>1$, and let $f_{0} \in S\left(B_{C}^{\prime}\right)$ which satisfies that $f_{0}(x)=\|x\|=\sup _{f \in S\left(B_{C}^{\prime}\right)} f(x)$.

First we consider the points on the open segment $\left(x^{\prime}, x\right)$ where $x^{\prime}$ is the intersect point, different from $x$ of $\operatorname{bd}(\hat{C}(x))$ (where bd denotes the boundary) and the line generated by $o$ and $x$. For any $y \in\left(x^{\prime}, x\right)$, there exists a $\mu$ with $-\|x\|^{-1}<\mu<1$ such that $y=\mu x$.

Hence we have

$$
\sup _{f \in F_{C}^{1}} \frac{1-f(y)}{1+f(y)}=\sup _{f \in F_{C}^{1}} \frac{1-\mu f(x)}{1+\mu f(x)}=\max \left\{\frac{1-\mu}{1+\mu}, \frac{1+\mu}{1-\mu}\right\}
$$

since the function $g_{1}(s):=(1-\mu s) /(1+\mu s)\left(-\|x\|^{-1}<\mu<1\right)$ is strictly increasing or decreasing on $s \in[-1,1]$ and there exist $f_{1}, f_{2} \in F_{C}^{1}$ such that $f_{1}(x)=1$ and 
$f_{2}(x)=-1$ (notice the construction of $\hat{C}(x)$ which guarantees the existence of the support hyperplanes, passing $x$, to $C$ ).

Furthermore,

$$
\sup _{f \in F_{C}^{2}} \frac{f(x)-f(y)}{1+f(y)}=\sup _{f \in F_{C}^{2}} \frac{(1-\mu) f(x)}{1+\mu f(x)}=\frac{(1-\mu)\|x\|}{1+\mu\|x\|}
$$

since the function $g_{2}(s):=(1-\mu) s /(1+\mu s)\left(-\|x\|^{-1}<\mu<1\right)$ is strictly increasing on $s \in[1, \infty)$ and $1 \leq f(x) \leq\|x\|$ for any $f \in F_{C}^{2}$, and $f_{0} \in F_{C}^{2}, f_{0}(x)=\|x\|$.

Similarly we have

$$
\sup _{f \in F_{C}^{2}} \frac{1+f(y)}{f(x)-f(y)}=\frac{1+\mu\|x\|}{(1-\mu)\|x\|} .
$$

Therefore we get

$$
r_{y}=\max \left\{\frac{1-\mu}{1+\mu}, \frac{1+\mu}{1-\mu}, \frac{(1-\mu)\|x\|}{1+\mu\|x\|}, \frac{1+\mu\|x\|}{(1-\mu)\|x\|}\right\} .
$$

Since when $\mu \in\left(-\|x\|^{-1}, 1\right)$,

$$
\begin{aligned}
g_{2}(1) & =\frac{1-\mu}{1+\mu} \leq g_{2}(\|x\|)=\frac{(1-\mu)\|x\|}{1+\mu\|x\|}, \\
\left(g_{2}(1)\right)^{-1} & =\frac{1+\mu}{1-\mu} \geq\left(g_{2}(\|x\|)\right)^{-1}=\frac{1+\mu\|x\|}{(1-\mu)\|x\|},
\end{aligned}
$$

we have

$$
r_{y}=\max \left\{\frac{1+\mu}{1-\mu}, \frac{(1-\mu)\|x\|}{1+\mu\|x\|}\right\} .
$$

Now notice that the function $g_{3}(\mu):=(1+\mu) /(1-\mu)$ is strictly increasing and $g_{4}(\mu):=(1-\mu)\|x\| /(1+\mu\|x\|)$ is strictly decreasing on $\left(-\|x\|^{-1}, 1\right)$, so if we let $\mu^{*}$ be the solution of the equation $g_{3}(\mu)=g_{4}(\mu)$, i.e., $\mu^{*}:=(\|x\|-1) /(3\|x\|+1)$, and set $x^{*}:=\mu^{*} x$, then

$$
r_{y}=\left\{\begin{array}{lll}
(1-\mu)\|x\| /(1+\mu\|x\|), & \text { if } & -\|x\|^{-1}<\mu \leq \mu^{*}, \\
(1+\mu) /(1-\mu), & \text { if } & \mu^{*}<\mu<1,
\end{array}\right.
$$

and

$$
\begin{aligned}
\min _{y \in\left(x^{\prime}, x\right)} r_{y} & =\min _{\mu \in\left(-\|x\|^{-1}, 1\right)}\left(\max \left\{\frac{1+\mu}{1-\mu}, \frac{(1-\mu)\|x\|}{1+\mu\|x\|}\right\}\right) \\
& =g_{3}\left(\mu^{*}\right)=g_{4}\left(\mu^{*}\right)=\frac{2\|x\|}{\|x\|+1} .
\end{aligned}
$$

To prove that $x^{*}$ is the (unique) critical point of $\hat{C}(x)$ and that as $(\hat{C}(x))=2\|x\| /$ $(\|x\|+1)$, we consider the set $\mu^{*} \hat{C}(x)$. 
First we show that for any $y \in \mu^{*} \hat{C}(x), f_{0}(y) \leq \mu^{*}\|x\|$ and $f_{0}(y)=\mu^{*}\|x\|$ iff $y=\mu^{*} x$. In fact since

$$
\hat{C}(x)=\left\{y: y=\lambda x+(1-\lambda) x_{1}, x_{1} \in C, 0 \leq \lambda \leq 1\right\},
$$

we know that

$$
\lambda^{*} \hat{C}(x)=\left\{y: y=\lambda x+\left(\mu^{*}-\lambda\right) x_{1}, x_{1} \in C, 0 \leq \lambda \leq \mu^{*}\right\},
$$

hence for any $y \in \mu^{*} \hat{C}(x)$, since $f_{0}\left(x_{1}\right) \leq 1<\|x\|$ for any $x_{1} \in C$,

$$
f_{0}(y)=\lambda f_{0}(x)+\left(\mu^{*}-\lambda\right) f_{0}\left(x_{1}\right) \leq \lambda\|x\|+\left(\mu^{*}-\lambda\right)\|x\|=\mu^{*}\|x\|,
$$

and $f_{0}(y)=\mu^{*}\|x\|$ iff $\lambda=\mu^{*}$, i.e., $y=\mu^{*} x$.

Now for any $y \in \operatorname{int}(\hat{C}(x))$, if $y \in \mu^{*} \hat{C}(x)$ and $y \neq \mu^{*} x$, then by what was just shown above, there exists a $\mu<\mu^{*}$ such that $f_{0}(y)=\mu\|x\|$, so we get

$$
\begin{aligned}
r_{y} & \geq \sup _{f \in F_{C}^{2}} \frac{f(x)-f(y)}{1+f(y)} \geq \frac{f_{0}(x)-f_{0}(y)}{1+f_{0}(y)} \\
& =\frac{(1-\mu)\|x\|}{1+\mu\|x\|}>\frac{\left(1-\mu^{*}\right)\|x\|}{1+\mu^{*}\|x\|}=g_{4}\left(x^{*}\right)=\frac{2\|x\|}{\|x\|+1} .
\end{aligned}
$$

If $y \notin \mu^{*} \hat{C}$, then by the fact that

$$
\hat{C}(x)=\left\{y:|f(y)| \leq 1, f \in F_{C}^{1}\right\},
$$

and so

$$
\mu^{*} \hat{C}(x)=\left\{y:|f(y)| \leq \mu^{*}, f \in F_{C}^{1}\right\},
$$

we know that there exists a $f_{1} \in F_{C}^{1}$ such that $\left|f_{1}(y)\right|=\mu>\mu^{*}$, therefore

$$
\begin{aligned}
r_{y} & \geq \sup _{f \in F_{C}^{1}} \frac{1-f(y)}{1+f(y)} \geq \frac{1+\left|f_{1}(y)\right|}{1-\left|f_{1}(y)\right|} \\
& =\frac{1+\mu}{1-\mu}>\frac{1+\mu^{*}}{1-\mu^{*}}=g_{3}\left(\mu^{*}\right)=\frac{2\|x\|}{\|x\|+1} .
\end{aligned}
$$

The proof is finished.

Remark 2. By Theorem 1 and Fact 2, we can easily get the asymmetry functions of general symmetric convex bodies:

For a symmetric convex body $C \subset R^{n}$ with $x_{0}$ as its center, we have

$$
A_{C}(x)=\max \left\{1, \frac{2\left\|x-x_{0}\right\|_{C}}{\left\|x-x_{0}\right\|_{C}+1}\right\}, \quad x \in R^{n},
$$

where $\|\cdot\|_{C}$ denotes the norm of Banach space $R^{n}$ with $C-x_{0}$ as its unit ball.

The following corollaries can be deduced from Theorem 1 immediately. 


\section{Corollary 1.}

(1) For any $n, m \geq 2$, any two symmetric convex bodies $C \subset R^{n}$ with center at $x_{C}$, $D \subset R^{m}$ with center at $y_{D}$, if $x \in R^{n}, y \in R^{m}$ such that $\left\|x-x_{C}\right\|_{C}=\left\|y-y_{D}\right\|_{D}$, then $A_{C}(x)=A_{D}(y)$.

(2) For a symmetric convex body $C \subset R^{n}$, if $x, y \in R^{n}$ satisfy that $\|x\|_{C}=\|y\|_{C}$, then $A s(x)=A s(y)$.

Corollary 2. For a symmetric convex body $C \subset R^{n}$, the following are true:

(1) For $\left.1<\lambda<2,\left\{x: A_{C}(x)\right\}=\lambda\right\}=\operatorname{bd}(\hat{\lambda} C)$, and for $1 \leq \lambda<2,\left\{x, A_{C}(x) \leq\right.$ $\lambda\}=\hat{\lambda} C$, where $\hat{\lambda}=\lambda /(2-\lambda)$ and the homothetic center is the center of $C$.

(2) For $1 \leq \lambda<2,\left\{x^{*}: A_{C}(x)=\lambda\right\}=\operatorname{bd}(\check{\lambda} C)$, and $\left\{x^{*}: A_{C}(x) \leq \lambda\right\}=\lambda^{*} C$, where $x^{*}$ denotes the critical point of $\hat{C}(x), \grave{\lambda}=((\lambda-1) /(\lambda+1)) \hat{\lambda}$ and the homothetic center is the center of $C$ as well.

\section{Asymmetry Functions of $(n-1)$-Dimensional Convex Bodies}

For non-symmetric convex bodies, as is to be expected, the asymmetry functions become more complicated and more interesting. However, we leave this general topic to other papers. Here we consider the general cones by which we mean the convex hull of a single point and some convex body of dimension $n-1$.

Let $H \subset R^{n}$ be a hyperplane, and let $D \subset H$ be an $(n-1)$-dimensional convex body. For convenience, denote by $F_{D}^{(n-1)}$ the set of all affine functions $g$ on $H$ such that $\{g=1\},\{g=-1\}$ are both support hyperplanes to $D$ in $H$, and note

$$
r_{y}^{H}=\sup _{g \in F_{D}^{(n-1)}} \frac{1-g(y)}{1+g(y)} \quad \text { for } \quad y \in \operatorname{reint}(D),
$$

where reint denotes the relative interiors of $D$, and still denote by as $(D)$ the asymmetry number of $D$ with respect to $H$.

Now set, for any $z \in R^{n} \backslash H, \hat{D}_{z}=\operatorname{conv}(z, D)$ which is obviously a cone, and denote as $\left(\hat{D}_{z}\right)$ by $A_{D}(z)$ as well, then we have the following:

Theorem 2. For any $z \in R^{n} \backslash H, A_{D}(z)=\operatorname{as}(D)+1$, and all the critical points $x^{*}$ of $\hat{D}_{z}$ have the form

$$
x^{*}=\frac{1}{2+\operatorname{as}(D)} z+\left(\frac{1+\operatorname{as}(D)}{2+\operatorname{as}(D)}\right) x^{\prime},
$$

where $x^{\prime}$ is a critical point of $D$.

Proof. First we prove a general fact that if $x^{\prime} \in \operatorname{reint}(D)$ with $r_{x^{\prime}}^{H}=d$, then

$$
\min \left\{r_{y} ; y \in\left(x^{\prime}, z\right)\right\}=r_{x_{0}}=d+1,
$$

where $\left(x^{\prime}, z\right)=\left\{y ; \quad y=\lambda z+(1-\lambda) x^{\prime}, 0<\lambda<1\right\}$, and $x_{0}=(1 /(2+d)) z+$ $((1+d) /(2+d)) x^{\prime}$. 
To do this, we first show that $r_{x_{0}}=d+1$,

For any $f \in F_{\hat{D}_{z}}$, if $f_{\mid H} \in F_{D}^{(n-1)}$ (where $f_{\mid H}$ denotes the restriction of $f$ on $H$ ), by $\left(1-f_{\mid H}\left(x^{\prime}\right)\right) /\left(1+f_{\mid H}\left(x^{\prime}\right)\right) \leq d$, i.e., $f\left(x^{\prime}\right)=f_{\mid H}\left(x^{\prime}\right) \geq(1-d) /(1+d)$, we have

$$
f\left(x_{0}\right)=\frac{1}{2+d} f(z)+\frac{1+d}{2+d} f\left(x^{\prime}\right) \geq \frac{1}{2+d}(-1)+\frac{1+d}{2+d} \frac{1-d}{1+d}=\frac{-d}{2+d},
$$

therefore

$$
\frac{1-f\left(x_{0}\right)}{1+f\left(x_{0}\right)} \leq \frac{1-(-d /(2+d))}{1+(-d /(2+d))}=d+1 .
$$

If $f_{\mid H} \notin F_{C}^{(n-1)}$, then at least one of $\left\{f_{\mid H}=1\right\},\left\{f_{\mid H}=-1\right\}$ is not the support hyperplane to $D$. Suppose both are not, then by the construction of $\hat{D}_{z}$, it is clear that $f= \pm f^{*}$ where $f^{*}$ is the affine functional satisfying that $f^{*}(z)=1, f^{*}(H)=-1$. In the case when $f=f^{*}$,

$$
f\left(x_{0}\right)=\frac{1}{2+d} f^{*}(z)+\frac{1+d}{2+d} f^{*}\left(x^{\prime}\right)=\frac{1}{2+d}+\frac{1+d}{2+d}(-1)=\frac{-d}{2+d}
$$

and hence

$$
\frac{1-f\left(x_{0}\right)}{1+f\left(x_{0}\right)}=\frac{1-(-d /(2+d))}{1+(-d /(2+d))}=d+1
$$

When $f=-f^{*}$, then

$$
f\left(x_{0}\right)=\frac{d}{2+d}, \frac{1-f\left(x_{0}\right)}{1+f\left(x_{0}\right)}=\frac{1}{d+1} \leq d+1 .
$$

Now suppose $\left\{f_{\mid H}=-1\right\}$ is the only support hyperplane to $D$, then $f(z)=1$, so

$$
\begin{aligned}
f\left(x_{0}\right) & =\frac{1}{2+d} f(z)+\frac{1+d}{2+d} f\left(x^{\prime}\right) \\
& \geq \frac{1}{2+d}+\left(1-\frac{1}{2+d}\right)(-1)=\frac{-d}{2+d}
\end{aligned}
$$

and $\left(1-f\left(x_{0}\right)\right) /\left(1+f\left(x_{0}\right)\right) \leq d+1$.

While $\left\{f_{\mid H}=1\right\}$ is the only support hyperplane to $D$ (then $f(z)=-1$ ), we choose a $g \in F_{D}^{(n-1)}$ such that $\{g=1\}=\left\{f_{\mid H}=1\right\}$. Notice that for any $u \in\left\{f_{\mid H}=-1\right\}, g(u) \leq$ $f(u)$, we could easily prove that $g\left(x^{\prime}\right) \leq f_{\mid H}\left(x^{\prime}\right)=f\left(x^{\prime}\right)$. Now choose $\bar{f} \in F_{\hat{D}_{z}}$ such that $\bar{f}(z)=f(z)=-1, \bar{f}_{\mid H}=g$. Then

$$
\bar{f}\left(x_{0}\right)=\frac{1}{2+d} \bar{f}(z)+\frac{1+d}{2+d} \bar{f}\left(x^{\prime}\right) \leq \frac{1}{2+d} f(z)+\frac{1+d}{2+d} f\left(x^{\prime}\right)=f\left(x_{0}\right) .
$$

So by $\bar{f}_{\mid H}=g \in F_{D}^{(n-1)}$, we have

$$
\frac{1-f\left(x_{0}\right)}{1+f\left(x_{0}\right)} \leq \frac{1-\bar{f}\left(x_{0}\right)}{1+\bar{f}\left(x_{0}\right)} \leq d+1 .
$$


Finally we get (notice that $\left.\left(1-f^{*}\left(x_{0}\right)\right) /\left(1+f^{*}\left(x_{0}\right)\right)=d+1\right)$

$$
r_{x_{0}}=\sup _{f \in F_{\hat{D}_{z}}} \frac{1-f\left(x_{0}\right)}{1+f\left(x_{0}\right)}=d+1 .
$$

Now for other $y \in\left(x^{\prime}, z\right)$, there is a $0<\lambda<1, \lambda \neq 1 /(2+d)$ such that $y=$ $\lambda z+(1-\lambda) x^{\prime}$.

If $\lambda<1 /(2+d)$, then $f^{*}(y)=\lambda f^{*}(z)+(1-\lambda) f^{*}\left(x^{\prime}\right)=2 \lambda-1<2(1 /(2+d))-$ $1=-d /(2+d)$, so

$$
r_{y} \geq \frac{1-f^{*}(y)}{1+f^{*}(y)}>\frac{1-(-d /(2+d))}{1+(-d /(2+d))}=d+1
$$

If $\lambda>1 /(2+d)$, we choose $\tilde{f} \in F_{\hat{D}_{z}}$ such that $\tilde{f}(z)=-1, \tilde{f}_{\mid H}=g^{*}$, where $g^{*} \in$ $F_{D}^{(n-1)}$ satisfies that $\left(1-g^{*}\left(x^{\prime}\right)\right) /\left(1+g^{*}\left(x^{\prime}\right)\right)=d$, i.e., $g^{*}\left(x^{\prime}\right)=(1-d) /(1+d)$. Then

$$
\begin{aligned}
\tilde{f}(y) & =\lambda \tilde{f}(z)+(1-\lambda) \tilde{f}\left(x^{\prime}\right)<\frac{1}{2+d} \tilde{f}(z)+\left(1-\frac{1}{2+d}\right) \tilde{f}\left(x^{\prime}\right) \\
& =\frac{1}{2+d}(-1)+\left(1-\frac{1}{2+d}\right) \frac{1-d}{1+d}=\frac{-d}{2+d},
\end{aligned}
$$

so again

$$
r_{y} \geq \frac{1-\tilde{f}(y)}{1+\tilde{f}(y)}>\frac{1-(-d /(2+d))}{1+(-d /(2+d))}=d+1 .
$$

We proved what we claimed.

Now it follows easily that

$$
A_{D}(z)=\operatorname{as}\left(\hat{D}_{z}\right)=\inf _{x^{\prime} \in \operatorname{reint}(D)}\left\{r_{x^{\prime}}+1\right\}=\operatorname{as}(D)+1
$$

and $x^{*}=(1 /(2+d)) z+((1+d) /(2+d)) x^{\prime}$ is a critical point of $\hat{D}_{z}$ iff $x^{\prime}$ is a critical point of $D$. The proof of the theorem is now completed.

Remark 3. From the proof we know that $\hat{D}(x)$ is of unique critical point iff $D$ is of unique critical point.

\section{Acknowledgment}

The authors express their thanks to the referee's comments which strengthened the paper. 


\section{References}

1. A. S. Besicovitch, Asymmetry of a plan convex set with respect to its centroid, Pacific J. Math. 8 (1958), 335-337.

2. G. D. Chakerian, S. K. Stein, On the measures of symmetry of convex bodies, Canad. J. Math. 17 (1965), 497-504.

3. H. T. Croft, K. J. Falconer, R. K. Guy (editors), Unsolved Problems in Geometry, Springer-Verlag, New York, 1991.

4. H. Groemer, Stability theorems for two measures of symmetry, Discrete Comput. Geom. 24 (2000), 301311.

5. H. Groemer, Stability of geometric inequalities, Handbook of Convex Geometry, North-Holland, Amsterdam, 1993, Section 1.4.

6. B. Grünbaum, Measure of symmetry of convex sets, in Convexity, Proceedings of Symposia in Pure Mathematics 7, American Mathematical Society, Providence, 1963, pp. 233-270.

7. S. Kaijser, Q. Guo, An estimate of the affine distance between convex bodies (Preprint), U.U.D.M. Report 1992:16.

8. S. Kaijser, Q. Guo, Approximation of convex bodies by convex bodies (Preprint), U.U.D.M. Report 2000:23.

9. V. L. Klee, Jr, The critical set of a convex set, Amer. J. Math. 75 (1953), 178-188.

Received March 1, 2001, and in revised form June 29, 2001. Online publication December 17, 2001. 